\title{
Identifying conserved molecular targets required for cell migration of glioblastoma cancer stem cells
}

Josephine Volovetz ${ }^{1,2}$, Artem D. Berezovsky ${ }^{1}$, Tyler Alban ${ }^{1,2}$, Yujun Chen ${ }^{3}$, Adam Lauko ${ }^{1,2}$, George F. Aranjuez, ${ }^{1,6}$, Ashley Burtscher ${ }^{1}$, Kelly Shibuya ${ }^{1,2}$, Daniel J. Silver', John Peterson', Danny Manor ${ }^{4}$, Jocelyn A. McDonald (10) ${ }^{1,3}$ and Justin D. Lathia (1D) $1,2,5$

\begin{abstract}
Glioblastoma (GBM) is the most prevalent primary malignant brain tumor and is associated with extensive tumor cell infiltration into the adjacent brain parenchyma. However, there are limited targeted therapies that address this disease hallmark. While the invasive capacity of self-renewing cancer stem cells (CSCs) and their non-CSC progeny has been investigated, the mode(s) of migration used by CSCs during invasion is currently unknown. Here we used time-lapse microscopy to evaluate the migratory behavior of CSCs, with a focus on identifying key regulators of migration. A head-to-head migration assay demonstrated that CSCs are more invasive than non-CSCs. Time-lapse live cell imaging further revealed that GBM patient-derived CSC models either migrate in a collective manner or in a single cell fashion. To uncover conserved molecular regulators responsible for collective cell invasion, we utilized the genetically tractable Drosophila border cell collective migration model. Candidates for functional studies were generated using results from a targeted Drosophila genetic screen followed by gene expression analysis of the human homologs in GBM tumors and associated GBM patient prognosis. This strategy identified the highly conserved small GTPase, Rap1a, as a potential regulator of cell invasion. Alteration of Rapla activity impaired the forward progress of Drosophila border cells during development. Rap1a expression was elevated in GBM and associated with higher tumor grade.

Functionally, the levels of activated Rap1a impacted CSC migration speed out of spheres onto extracellular matrix. The data presented here demonstrate that CSCS are more invasive than non-CSCs, are capable of both collective and single cell migration, and express conserved genes that are required for migration and invasion. Using this integrated approach, we identified a new role for Rapla in the migration of GBM CSCs.
\end{abstract}

\section{Introduction}

Glioblastoma (GBM), the most prevalent primary malignant brain tumor in adults, remains one of the most lethal cancers, with a median survival of less than 2 years ${ }^{1-4}$. Barriers to effective treatment include the extensive

\footnotetext{
Correspondence: Jocelyn A. McDonald (jmcdona@ksu.edu) or

Justin D. Lathia (lathiaj@ccf.org)

'Lerner Research Institute, Cleveland Clinic, Cleveland, OH 44195, USA

${ }^{2}$ Cleveland Clinic Lerner College of Medicine of Case Western Reserve

University, Cleveland, OH 44195, USA

Full list of author information is available at the end of the article

These authors contributed equally: Josephine Volovetz, Artem D. Berezovsky, Tyler Alban

These authors jointly supervised this work: Jocelyn A. McDonald,

Justin D. Lathia

Edited by I. Amelio
}

infiltration of tumor cells throughout the brain and the high degree of inter- and intra-tumoral heterogeneity. GBM also contains self-renewing cancer stem cell (CSC) populations that are resistant to conventional therapies ${ }^{5,6}$. While many studies have identified mechanisms through which CSCs expand and are resistant to radiation and Temozolomide, the standard of care chemotherapy, less is known about the mechanisms that drive invasion. Several studies have reported that CSCs display elevated invasive potential over non- $\mathrm{CSCs}^{7-9}$. However, the differences between CSC and non-CSC invasion are unclear, as previous assessments have been done by comparing cell populations in isolation. A number of pathways drive GBM cell invasion, such as the Wingless/Int1 (Wnt) and TGF- $\beta$ pathways ${ }^{10}$. Since these

\section{(c) The Author(s) 2020}

\footnotetext{
(c) Open Access This article is licensed under a Creative Commons Attribution 4.0 International License, which permits use, sharing, adaptation, distribution and reproduction cc) in any medium or format, as long as you give appropriate credit to the original author(s) and the source, provide a link to the Creative Commons license, and indicate if changes were made. The images or other third party material in this article are included in the article's Creative Commons license, unless indicated otherwise in a credit line to the material. If material is not included in the article's Creative Commons license and your intended use is not permitted by statutory regulation or exceeds the permitted use, you will need to obtain permission directly from the copyright holder. To view a copy of this license, visit http://creativecommons.org/licenses/by/4.0/.
} 
mechanisms are also associated with self-renewal ${ }^{11}$, the extent to which they drive invasion over self-renewal has yet to be determined. Increased invasion has been observed with anti-angiogenic therapies ${ }^{12,13}$. Additionally, GBM cells visualized in vivo tend to migrate along the luminal surface of blood vessels, white matter tracts, and the subarachnoid space below the meningeal covering of the brain and the brain parenchyma ${ }^{14}$. Studies in the perivascular environment revealed that cells migrate faster and induce further microvascular development ${ }^{15,16}$. These observations suggest that invasion itself may contribute to therapeutic resistance in GBM.

The mode by which GBM cells, particularly CSCs, migrate and invade the brain is poorly understood. Studies using tumor histology, live ex vivo tumor explants, and in vivo models demonstrate that cancer cells have the capacity to invade as single cells or as collectives, in which cells coordinate their movement by maintaining cell-cell contacts amongst small to large groups of cells ${ }^{17,18}$. Both single cell and collective cell modes of invasion have been observed in a multiple human tumors, including breast, thyroid, and lung carcinomas ${ }^{19-24}$. Additionally, migrating cancer cells are highly dynamic and can invade as single cells, loosely associated multicellular streams, collective nests or strands, or through expansive growth, with some cells changing their migration mode during movement in response to the surrounding tissue microenvironment ${ }^{17,25}$. Recent work has demonstrated that GBM cells can migrate as both single cells and as multicellular collectives, which may influence their capacity to infiltrate the surrounding brain parenchyma ${ }^{26,27}$. However, whether GBM CSCs themselves migrate and invade as cell collectives, and whether this differs from nonCSCs, has yet to be determined and is the focus of our studies.

Although collective cell invasion contributes to cancer, much of our current mechanistic understanding of how cells migrate as collective groups have been obtained by studying cells that move during normal development. Collective cell migration is a frequent mode in the embryo, where it contributes to the shaping and forming of many organs. Key examples include gastrulation to form the three embryonic germ layers, neural crest migration to give rise to craniofacial structures and the peripheral nervous system, zebrafish lateral line organ formation, and branching morphogenesis to elaborate tubular structures within organs (e.g. the mammary gland and mammalian lung) ${ }^{28,29}$. One of the best-studied models of developmental collective cell migration is Drosophila border cells, which migrate as a cohesive group of six to ten cells in the egg chamber, the functional unit of the ovary ${ }^{29}$. The border cell cluster migrates during oogenesis in two phases, both of which respond to specific ligands secreted by the oocyte: in the posterior phase, border cells undergo a long-range movement from the anterior end of the egg chamber to the oocyte at the posterior; in the dorsal phase, the cells undergo shortrange migration along the oocyte towards the dorsalanterior side of the egg chamber ${ }^{29,30}$. The ability to genetically manipulate and observe border cell migration in its native tissue environment in real time makes it a powerful tool for identifying conserved regulators of collective invasion in development and in cancer ${ }^{29,31,32}$. Moreover, the use of the Drosophila system has also recently been leveraged for studies to identify conserved molecular mechanisms that drive GBM cell proliferation, survival, and self-renewal ${ }^{33-35}$.

Here, we observed that GBM CSC models that migrate as collectives, individual cells or mixtures of both modes. Further, we used results from a Drosophila border cell screen to identify conserved genes that control cell migration, which represent potential targetable regulators of GBM CSC invasion. This approach identified Rap1a as a putative regulator. We found that human Rap1a levels were elevated in GBM, and altered Rapla activity impacted CSC migration. These data demonstrate the ability to identify molecular regulators of migration and invasion of GBM CSCs, including Rapla, using a multisystem approach.

\section{Results \\ CSCs are more invasive than non-CSCs}

Previous studies suggest CSCs have increased migration and invasion capacity compared to non-CSCs. However, these analyses were done separately and not in a competition assay that would normalize for confounding factors (e.g. media conditions or paracrine/autocrine factors). Therefore, we compared differentially labeled CSCs and non-CSCs in a head-to-head co-culture ECMbased cell invasion assay (Fig. 1a). We used an approach previously shown to assess breast cancer co-culture invasion $^{36}$. We labeled CSCs and non-CSCs, then seeded them and overlaid the cells with a 3D extracellular matrix. We then added a chemoattractant on top. Using this system, we compared patient-derived GBM CSC models (T387, T4121, and T3691), versus their corresponding non-CSC progeny, which were independently derived from patient-derived xenograft (PDX) models. After $24 \mathrm{~h}$, we assessed the extent of invasion into the matrix along the chemokine gradient via confocal imaging. In all models, we observed significantly more invasion by CSCs compared to non-CSCs (Fig. 1b, c). CSCs exhibited 2- to 5-fold increase in migration versus nonCSCs. These results thus demonstrate that CSCs are more invasive than non-CSCs when compared in identical conditions. 

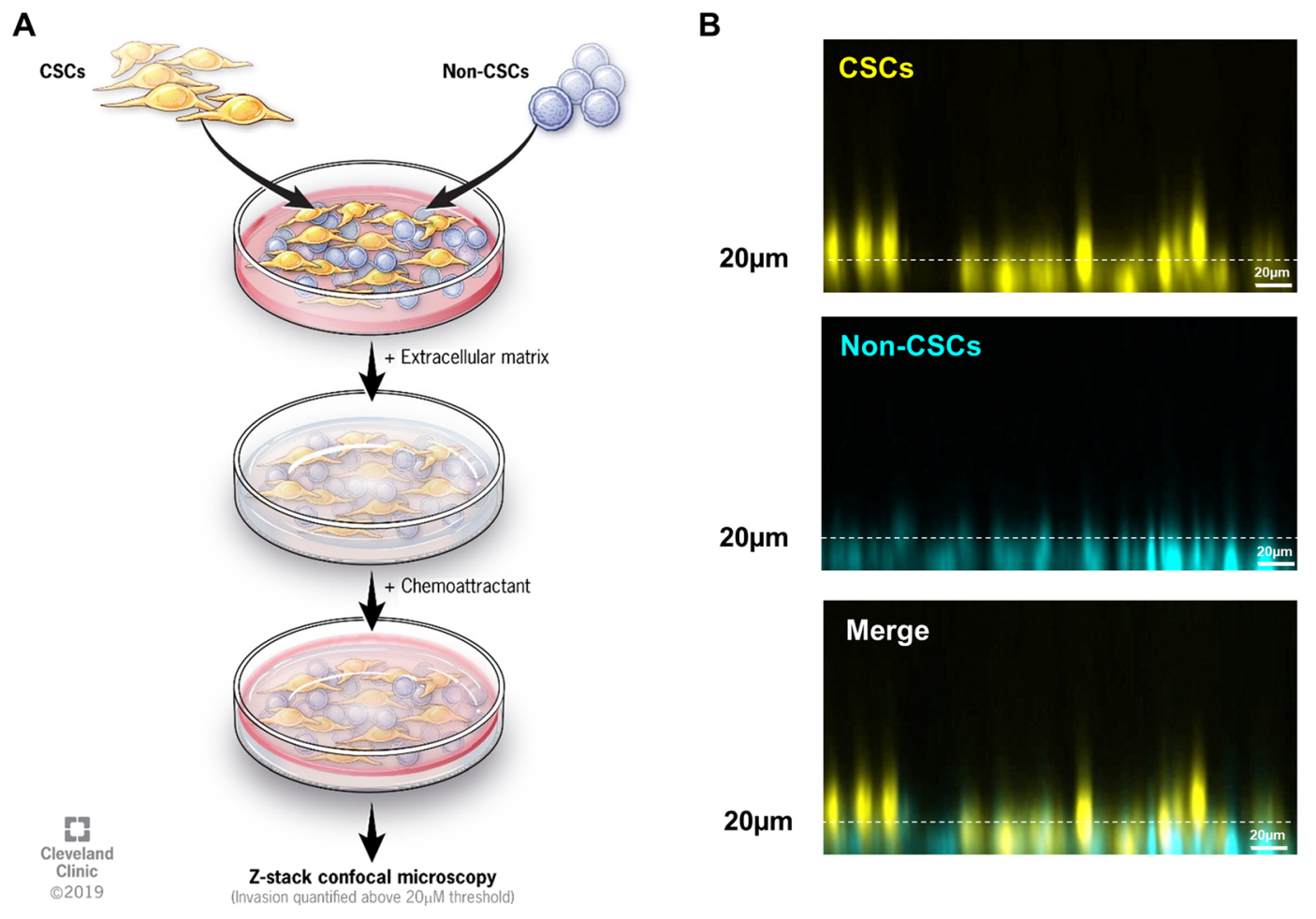

C

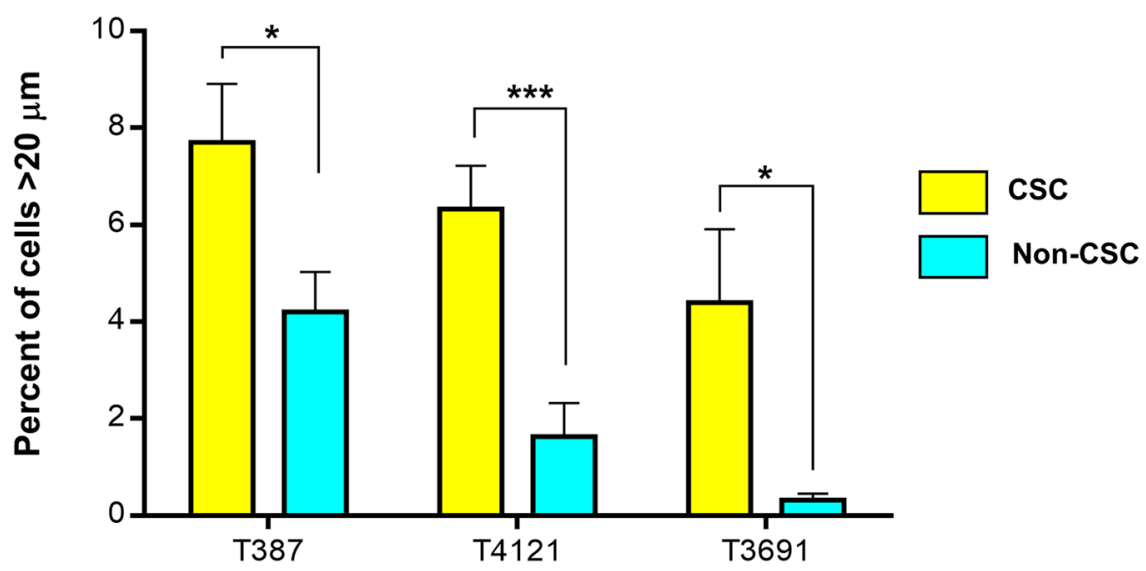

Fig. 1 Head to head migration of cancer stem cell and non-cancer stem cells. Schematic representation of the head-to-head migration assay of cancer stem cells (CSCS) and non-CSCs embedded into a 3D Geltrex extracellular matrix with a chemoattractant layered on top (a). Representative confocal z-stack projections of CSCs and non-CSCs $12 \mathrm{~h}$ post-seeding (CSCs = yellow, non-CSCs = blue) (b). Extent of migration of CSCs and nonCSCs from the bottom of the well (c). Statistics calculated based on an unpaired student's $t$ test, ${ }^{*} p<0.05,{ }^{* * *} p<0.001$.

Different CSC lines display different modes of migration

To assess how CSCs invade in terms of single cell and collective cell migration, we analyzed how CSCs exit a sphere culture. In this assay, eight separate CSC models were grown as spheres before being introduced to an extracellular matrix coated surface and then imaged using time-lapse microscopy. All CSCs were able to migrate away from the original sphere. Some models migrated individually (T4121), whereas others migrated as cell collectives, most often as cohesive "finger-like" projections or small nests (T387 and L1; Fig. 2, Supplemental Fig. 1). In the collectively migrating lines, the cells consistently stayed connected, with $\mathrm{N}$-cadherin enrichment at contacts between cells (Fig. 2, Supplemental Fig. 2). The majority of CSC models, displayed a mixture of migration modes, with cells moving away from the tumor sphere both as individuals and as collectives, most often in small cell groups (T3691, T3832, GBM10, L0, and T1919; Fig. 2, Supplemental Fig. 1). These data demonstrate that CSCs employ different modes of cell invasion, highlighting another 


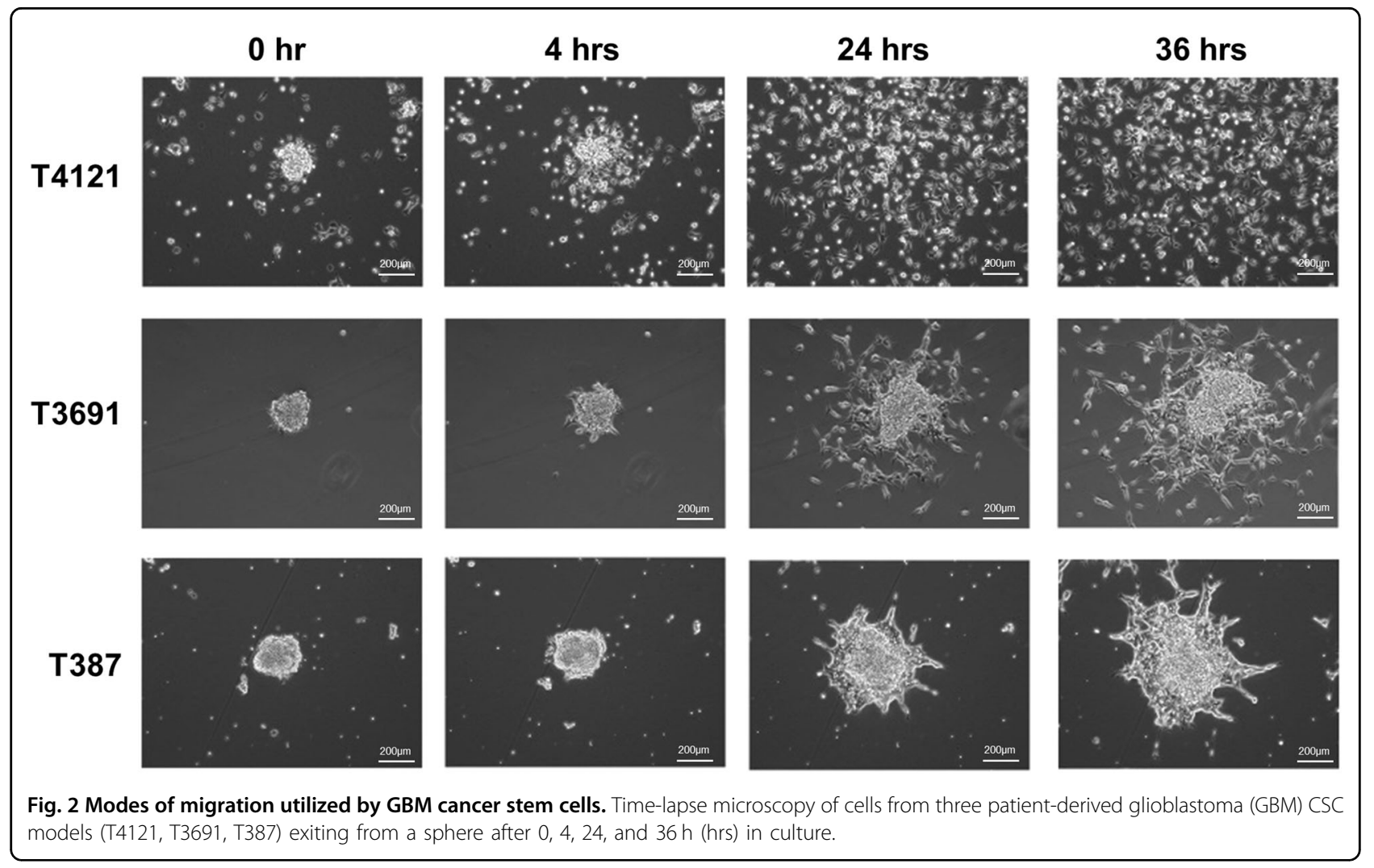

phenotype that displays inter-tumor and intra-tumor heterogeneity.

\section{Identification of candidate CSC invasion genes via border cell migration}

Given the observation that CSCs display different modes of cell invasion, we sought to determine the underlying mechanisms driving CSC migration patterns. Drosophila border cells represent a genetically tractable model of collective cell migration within an intact tissue. Many genes known to regulate border cell migration are highly conserved in humans and have been implicated in cancer $^{31,37,38}$. During mid-oogenesis, six to ten epithelial follicle cells are recruited to form the cohesive border cell cluster, which migrates as a coordinated unit over the course of about four hours towards the oocyte located at the posterior of the egg chamber ${ }^{39}$.

Recently, we performed an RNA interference (RNAi) screen targeting PDZ domain-containing genes to identify regulators of border cell collective migration ${ }^{40}$, which provided a starting point to identify candidate GBM migration genes. Proteins that contain the PDZ proteinprotein interaction domain facilitate the formation of multi-protein scaffolding complexes with conserved roles in signaling, cell polarization, and adhesion, making them excellent candidates to regulate the collective migration of normal and cancer cells. The majority of Drosophila
PDZ- (PSD95/Dlg/ZO1-) domain genes were screened for the ability to promote border cell migration ${ }^{40}$ (Fig. 3a). This screen identified high-confidence PDZ-domaincontaining genes (multiple transgenic RNAi lines targeting the gene were able to disrupt migration; Supplemental Table 1: Group 1 genes) and lower confidence genes (only one RNAi line per gene disrupted migration; Supplemental Table 1: Group 2 genes). We further included well-characterized genes known to promote border cell migration and/or interact with PDZ domain genes (Supplemental Table 1: Group 3 genes) ${ }^{30,37}$.

We identified human orthologs for these 40 Drosophila migration genes using FlyBase and the Drosophila RNAi Screening Center Integrative Ortholog Prediction Tool (DIOPT; http://www.flyrnai.org/diopt) ${ }^{41}$. Human orthologs were used to interrogate the TCGA-GBMLGG dataset in order to find overlapping genes that were relevant in both the migration model and glioma patients. Genes whose high expression correlated with decreased patient survival were compared to the list of genes that decreased border cell migration (Fig. 3b; Supplemental Table 1). Twenty-three genes fit these criteria and were candidates for further functional tests in border cells and GBM CSCs. Notably, genes encoding small GTPases, or targets of small GTPases, were well-represented (Fig. 3b). Small GTPases are known to promote both single cell and collective cell migration, and many interact with 
A

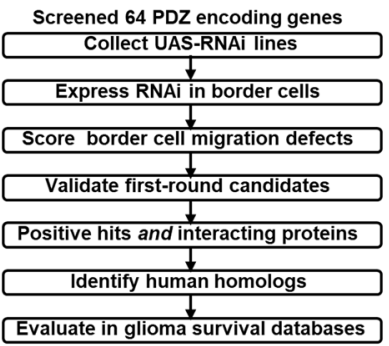

C

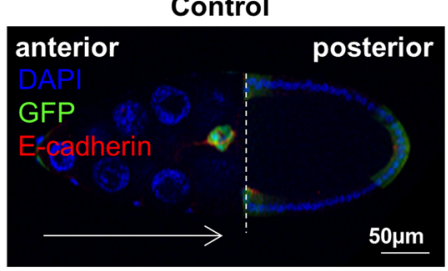

D

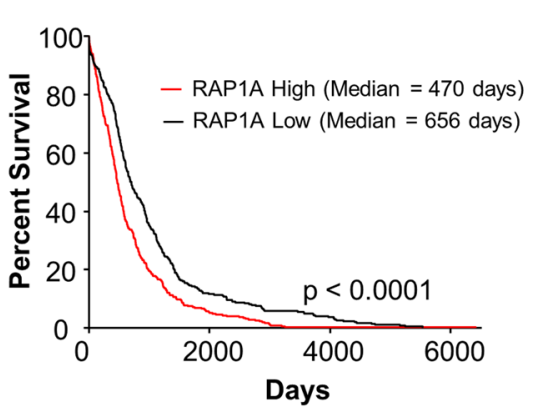

B

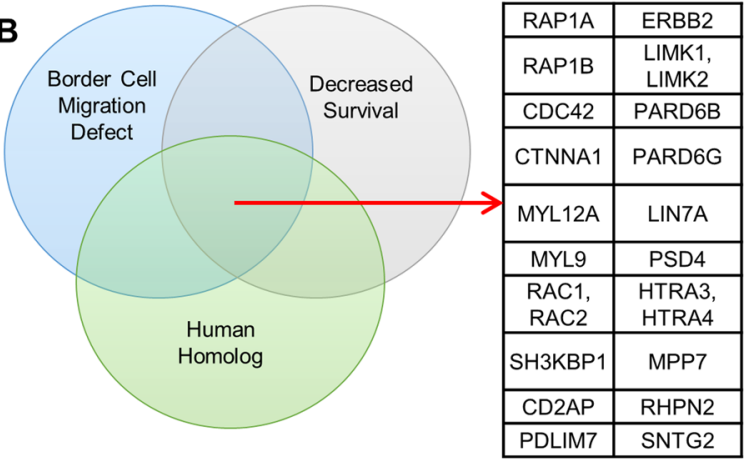

RAP1A Constitutively Active

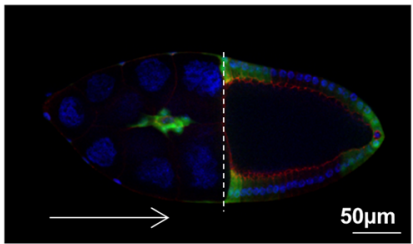

E

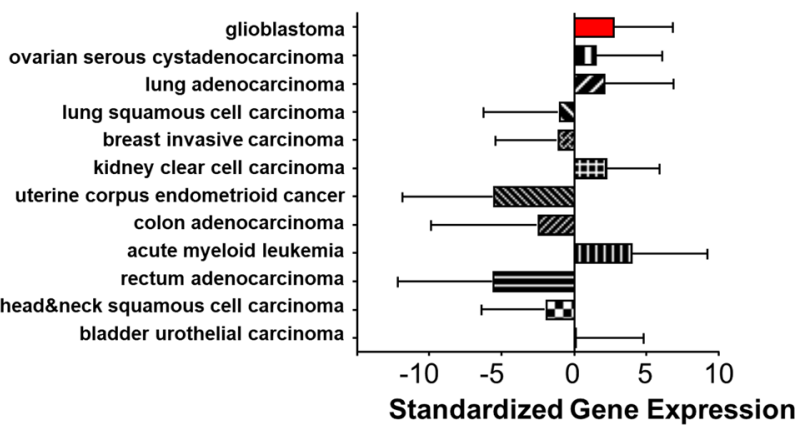

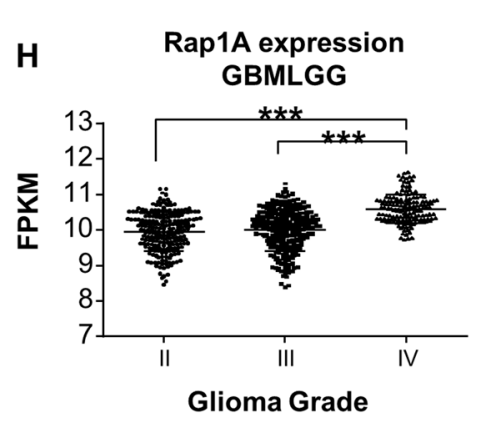

RAP1A expression (PANcan12 study)
RAP1A expression (GBMLGG) dataset

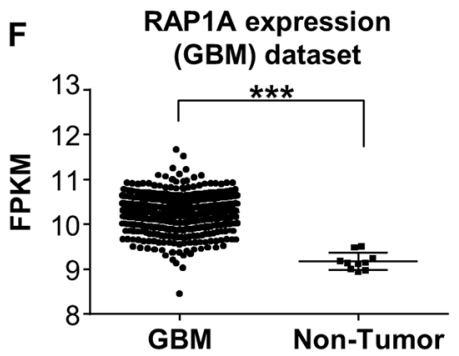

G

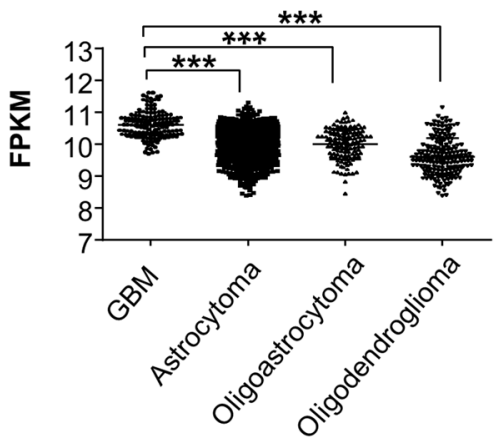

Fig. 3 Identification of Rap1a as a potential GBM invasion regulator. Flowchart of approach to identify human homologs of Drosophila genes that are required for migration and correlate with human glioma patient prognosis (a). Venn diagram of Drosophila genes involved in border cell migration, with annotated human homologs, and genes associated with decreased survival of GBM patients (b). Rap1a is one of twenty-three genes that meet these criteria. Control border cells (left panel, (c) in the Drosophila egg chamber collectively migrate towards the oocyte (dashed line; extent and direction of migration indicated by white arrow). Expression of constitutively active Rap1a (right panel, (c) results in failure to reach the oocyte and altered cluster shape. Border cells express UAS-mCD8:GFP (green) driven by slbo-GAL4. Egg chambers were immunostained for Ecadherin (red), which is highly expressed in border cells and other cell membranes. Nuclei were visualized using DAPI (blue). Evaluation of human Rap1a expression in the cancer genome atlas GBMLGG database and its association with glioma survival (d). Rap1a expression across the pan-cancer database of tumor mRNA expression demonstrates increased Rap1a in GBM and some other cancer types (e). Analysis of Rap1a expression in GBM versus non-tumor identified increased Rap1a expression in GBM tumors compared to non-tumors (f). Analysis of Rap1a expression in GBMLGG dataset identified increased expression in GBM tumors compared to other glioma tumors (g). Analysis of Rapla expression in GBMLGG dataset identified increased Rapla expression with increasing glioma grade (h). FPKM: fragments per kilobase of transcript per million mapped reads. Statistics calculated based on one-way ANOVA, ${ }^{* * *} p<0.001$ and differences in survival calculated based on log-rank analysis. 
PDZ-domain proteins ${ }^{42-44}$. For example, the highconfidence hit LIMK1 is downstream of Rac (human Rac1 and Rac2), which promotes membrane protrusions in migrating cells ${ }^{45,46}$. Similarly, Cdc42 stimulates actinrich protrusions and can polarize migrating cells through direct binding to Par-6 (human PARD6B/PARD6G), one of the strongest hits from the border cell screen ${ }^{47-49}$, and has a well-described role in GBM invasion ${ }^{50}$.

As a validation of candidate genes (Fig. 3a, b; Supplemental Table 1), we confirmed that the small GTPase Cdc42 was required for migration. In border cells, expression of a dominant-negative (DN) mutant form of Cdc42 (Cdc42 ${ }^{\mathrm{N} 17}$; DN Cdc42) severely blocked migration, with most border cells stopping along the migration pathway and failing to reach the oocyte (Supplemental Fig. 3a-c). Live border cells expressing DN Cdc42 overall had trouble initiating migration compared to controls (Supplemental Fig. 3c). Further, Cdc42 controlled migratory protrusions (Supplemental Fig. 3d). We found that DN Cdc42 border cell clusters extended more protrusions, and these protrusions were more persistent, with a longer lifetime than those produced by control border cells. These data are consistent with a recent study that found that Cdc42 promotes cell-cell communication amongst border cells, resulting in only one border cell at the front of the cluster being able to extend a productive protrusion, which facilitates migration of the entire collective to the oocyte ${ }^{51}$. Increasing the levels of Cdc42 via overexpression of wild-type (WT) Cdc42 also modestly impaired movement to the oocyte, although to a lesser extent than DN Cdc42 (Supplemental Fig. 3a, c). These results suggest that optimal levels of the $\mathrm{Cdc} 42$ small GTPase are required for migration in vivo as previously reported $^{51}$.

Similarly, in GBM, we reduced the activity of Cdc42 in T387 cells using a specific chemical inhibitor, ML $141^{52}$. Decreased Cdc42 reduced the cell migration out of a CSC sphere (Supplemental Fig. 4a), but not cell viability, survival or proliferation (Supplemental Fig. 4b-d). Our results are consistent with recent work that found that knockdown of Cdc42 by siRNA decreased GBM inva$\operatorname{sion}^{50}$. Further, constitutively active Cdc42 reduced survival in a PDX model of glioma, while increasing the invasive capacity in sphere exit assays.

\section{Rap1a levels are elevated in GBM patients and correlate with tumor grade}

Having established that this integrated approach can identify genes important for GBM CSC migration, we next focused on a candidate gene, human Rapla, which is less characterized in collective cell migration. We and others recently showed that inhibition of the Drosophila Rap1a homolog (Rap1) disrupted border cell migration to the oocyte due to defects in actin-rich protrusions and altered cell-cell adhesion ${ }^{53,54}$. Rap1a is also regulated by the screen multi-hit gene, PDZ-GEF ${ }^{40}$ (ortholog of human RapGEF2/PDZ-GEF1 and RapGEF6/PDZ-GEF2; Supplementary Table 1). Further, expression of constitutively active (CA) Rap1a impaired movement of the border cells to the oocyte (Fig. 3c) ) $^{53,54}$. Thus, similar to Cdc42, having the proper levels of Rapla is critical for collective migration of border cells. In GBM, Rap1a expression was increased compared to non-tumor tissue using the TCGA database (Fig. 3f). Increased Rap1a expression also correlated with GBM compared to other glioma tumors (Fig. $3 \mathrm{~g}$ ), as well as increasing tumor grade (Fig. $3 \mathrm{~h}$ ). Additionally, when compared across the pan-cancer dataset, GBM had elevated levels of Rapla compared with other common cancers, although it was not ubiquitously increased across all cancers (Fig. 3e). Survival analysis in the GBMLGG dataset also revealed that Rapla expression levels correlate with a poor prognosis (Fig. 3d).

\section{Activated Rap1a alters migration of CSCs}

Assessment of Rap1a in CSCs compared to their nonCSC progeny across multiple models revealed that Rap1a was elevated in models that migrate collectively or as a mix of collective and single cells (T387, T3691, T3832; Fig. 4a). However, T4121, a model that migrated predominantly as single cells has lower Rap1A in CSCs compared on non-CSCs (Fig. 4a). On the basis that Rap1a levels are generally elevated in GBM, and that Drosophila Rap1 is required for collective migration of border cells (Fig. 3$)^{53}$, we determined how altering Rapla levels and/or activity impacted CSC migration. As described above, T387 and T3691 CSCs migrate as collective fingers and small clusters, while T4121 CSCs migrate as single cells (Fig. 2). Both increasing the levels of wild-type Rap1a (WT) and expressing the constitutively activated Rap1a (63E) mutant caused reduced migration in T387 and T3691when compared to the untransfected control. In comparison with overexpression of wild-type Rap1a, constitutively active Rap1a did not alter the migration rate in T387 but reduced the migration rate in T3691 (Fig. 4b-e). However, dominant-negative (N17) Rap1a induced a slight increase in migration rate compared to other overexpression conditions and had a similar migration rate to untransfected control conditions in T387 (Fig. 4d). In T3691, dominant-negative Rap1a had increased migration compared to constitutively active conditions and similar levels to wild-type overexpression conditions (Fig. 4e). T4121, which displayed single cell migration, was not impacted by modulation of Rap1a levels (Fig. 4f). These data suggest that levels of active Rap1a are critical for CSC migration in models that rely on a collective or mixed mode of cell migration. 


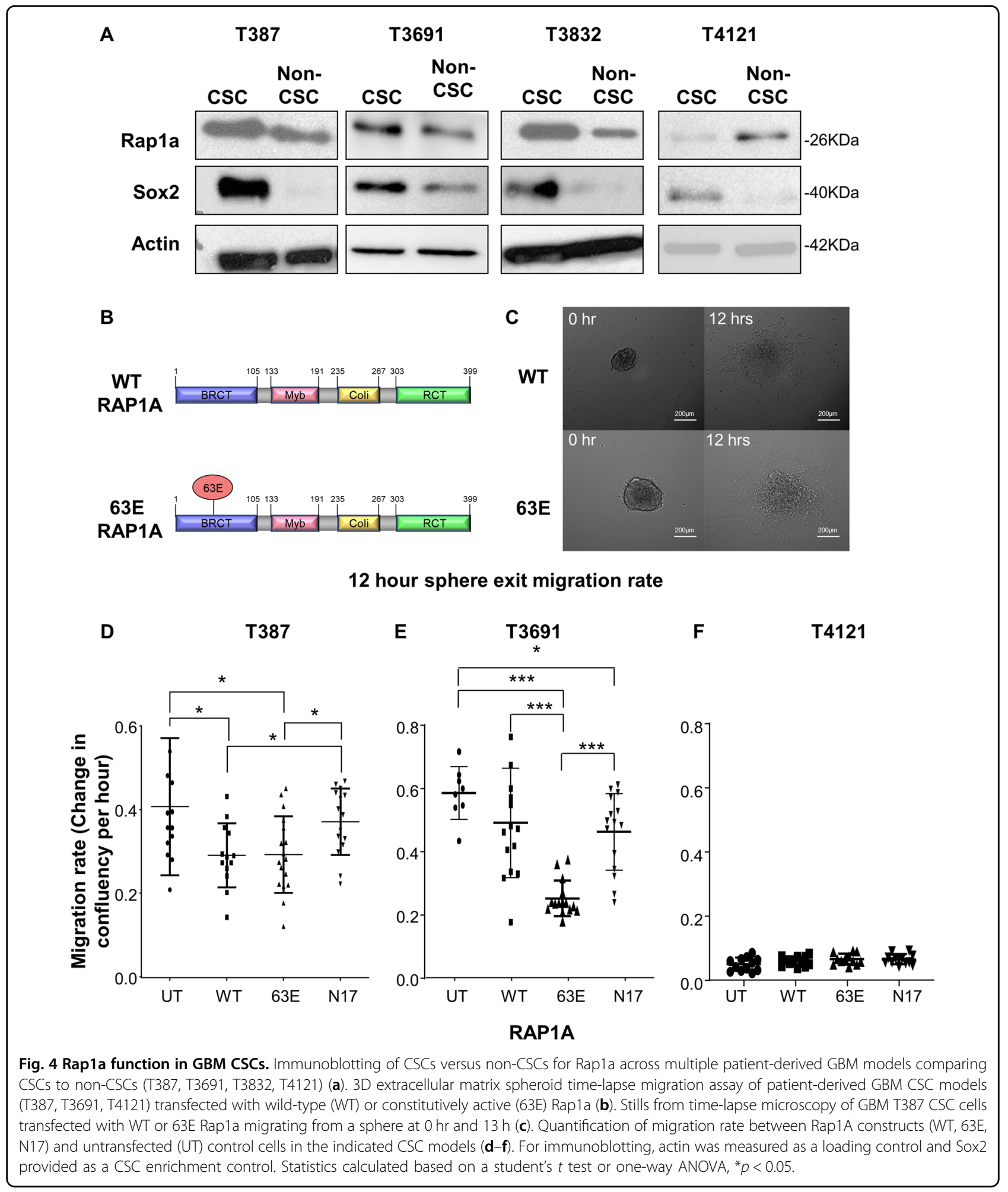

\section{Discussion}

Cancer cells migrate individually or as collectives ${ }^{18-20,29,55}$. The mode of migration may be critical for how well tumor cells, including those in GBM, invade the surrounding tissue and disseminate. Recent studies found that GBM cells can migrate collectively ${ }^{26,27}$, although whether GBM CSCs specifically used this type of migration was an open question. Our data indicate that a variety of patient-derived GBM CSC models can migrate individually and as mixtures of single cells and collectives. Interestingly, previous studies 
indicated that CSCs are likely more invasive than non-CSCs in $\mathrm{GBM}^{7-9}$. However, these analyses were done separately, so it was unknown whether CSCs could outcompete nonCSCs during invasion. Here, we directly compared the invasive capacity of CSCs to non-CSCs. Our results with this head-to-head assay allowed for greater control and comparison, and showed that CSCs are more invasive than nonCSCs. Currently it is unknown whether the mode of CSC invasion directly contributes to progression of GBM in patients. Recent work by the Friedl group demonstrated that human glioma cells can invade as multicellular groups, as well as individual cells, into 3D astrocyte scaffolds, mouse brain slice cultures, mouse brain xenografts, and in human tumor samples ${ }^{26,27}$. Specifically, glioma cells maintain cell-cell connections while moving along both blood vessels and the astrocyte-rich brain stroma ${ }^{26}$. Thus, it is likely that collective invasion of GBM CSCs helps disseminate tumors into the brain parenchyma and vasculature ${ }^{14}$, although this has yet to be directly tested.

As GBM invasion is a dynamic and multi-step process, the molecular mechanisms are still poorly understood. Our results highlight unique opportunities to apply knowledge about migration and invasion from model organisms to human diseases such as GBM. Border cell migration during Drosophila oogenesis has become a valuable tool for studying collective cell migration due to the wealth of genetic and cell biological methods that allow for wild-type and mutant cells to be imaged ex vivo while migrating within the native tissue ${ }^{56}$. We used an informed approach to identify genes required for border cell migration that may have a role in GBM. In particular, we focused on the highly conserved PDZ-domain encoding genes, many of which have known or suspected roles in human tumorigenesis and cancer cell invasion $^{57-59}$. Of these PDZ genes, plus related genes such as small GTPases with validated functions in border cells $^{30}$, we identified a number of conserved genes whose elevated expression correlated with decreased GBM patient survival. These results, as well as recent work by others, indicate that the small GTPases Cdc42 and Rap1A are needed for the migration of both border cells and GBM CSCs ${ }^{50,51,53,54}$. In addition, the differences in expression between CSCs and non-CSCs as well as functional manipulations suggest that Rap1A is operating more in collective migration as compared to single cell migration. Moreover, Rap1a expression is higher in the mesenchymal subtype (accessed via TCGA; data not shown), which has a high propensity for invasion ${ }^{60}$. Further work will be needed to determine the roles, if any, of the additional identified genes on GBM tumor growth, migration, and/or invasion. Given the high conservation of human disease genes in Drosophila, especially those implicated in tumor invasion and metastasis ${ }^{31}$, our results further suggest that border cell migration can be used to identify additional relevant regulators of GBM cancer cell migration and invasion.

Rap1a GTPase is known to regulate the migration of both normal cells and cancer cells ${ }^{61,62}$. Rap1 belongs to the Ras family of small GTPases, but is regulated by distinct guanine nucleotide exchange factors (GEFs) and GTPase activating proteins (GAPs), and generally has Ras-independent functions ${ }^{62}$. We found that constitutively active Rapla decreased the migration speed of CSCs compared to dominant-negative Rapla. However, elevated Rap1a levels correlate with worse GBM patient outcome. Additionally, Rap1 increases U87MG glioma spheroid invasion on collagen in response to plateletderived growth factor (PDGF) stimulation ${ }^{63}$. One way to reconcile these counterintuitive findings is to consider the possibility that Rap1a may have different roles in GBM CSCs versus non-CSCs, as well as unknown effects on tumor progression in vivo versus cell culture. Moreover, it is also possible that CSCs have an optimized level of Rap1a activity, and our overexpression conditions compromised this equilibrium. We hypothesize that speed and migration persistence could impact how tumors with elevated Rap1a expression invade the brain. Although we transiently increased Rap1a expression using a wild-type Rap1a construct and similarly observed slower migration, this may not reflect what happens in GBM tumors in the native environment. Chronic elevation of Rapla expression in GBM tumors could be worse for disease progression than transient activation. Interestingly, Rapla has been shown to be important for cellular proliferation in the context of in vivo GBM tumor growth ${ }^{64}$. It will be important to test whether tumors that overexpress Rap1a are more infiltrative in vivo, whether the main function of Rap1a in GBM is in cell proliferation ${ }^{64}$, or whether some combination of tumor growth, migration, and invasion is at play. The effect that Rap1a activity has on GBM patient survival is likely to be complex, and not simply one in which increased Rapla expression increases tumor invasion and thus decreases patient survival.

Overall, our data provide a rationale for additional studies on the role of Rap1a in GBM migration and invasion. Studies based on reducing cell migration and invasion should be a high priority due to the limited availability of anti-migration treatments, which remains difficult to target and is a driver of recurrence. Finally, our approach highlights the ability to leverage model organisms to identify key processes that drive GBM invasion and highlight a paradigm that could be applied to many other key cancer processes.

\section{Materials and methods Isolation of CSCs}

CSCs were isolated from surgically obtained adult GBM specimens and transiently amplified by implantation of 
unsorted tumor cells into immunocompromised mice as PDXs. All specimens were generated under approved Institutional Review Board protocols. For PDX dissociation, single cells were prepared from the bulk tumor by a Papain dissociation kit (Worthington Biochemical) as per the manufacturer's protocol and cultured using previously reported culturing methods ${ }^{65,66}$, with CSCs being enriched via CD133 beads (Miltenyi Biotec) and non-CSCs being devoid of CD133 cell surface expression. Cells were maintained in serum-free growth media supplemented with EGF and bFGF ("neurobasal complete media") as spheres or adherent on Geltrex solution (Thermo Fisher Scientific). The following PDX models, and their molecular subtype (if known), were used in this study: T387 (mesenchymal), T3832 (proneural), T3691 (proneural), T4121 (classical), T1919 (undetermined), L1 (undetermined), L0 (undetermined), and GBM10 (undetermined). All cell lines were authenticated by STR profiling and routinely tested for mycoplasma contamination.

\section{Head-to-head invasion assay}

The head-to-head invasion assay was adapted from Goswami et al. ${ }^{36}$ with minor modifications. First, cancer cells were stained with either Vybrant $\mathrm{DiO}$ or DiI cell labeling solutions (Thermo Fisher Scientific), according to manufacturer's directions. 150,000 to 200,000 cells in neurobasal complete media, from two distinct cell types, were added at 1:1 dilution to $35 \mathrm{~mm}$ glass bottom dishes (MatTek Corporation) that were coated with a dilute layer of Geltrex matrix. After $24 \mathrm{~h}$, media was removed and a thick, $80 \mu \mathrm{l}$ layer of Geltrex matrix was layered over the adherent cells. Dishes were returned to the incubator for $30 \mathrm{~min}$, in order for the matrix to solidify. Then, the dishes were flooded with $3 \mathrm{~mL}$ of neurobasal complete media. The following day, live cells were visualized using a PerkinElmer UltraView VOX Spinning Disk Confocal Imaging System on a Nikon DM1-6000 SD inverted microscope using a 10×/NA 0.7 air objective. A $100-\mu \mathrm{m}$ zstack image at $5-\mu \mathrm{m}$ increments was obtained. An extended $x-z$ projection was then obtained using the Volocity software (PerkinElmer) and invasion was quantified using Image ( $\mathrm{NIH}$, Bethesda, MD, USA) as the percentage of Vybrant dye signal found above $20 \mu \mathrm{m}$ from the bottom of the dish, compared with total fluorescence within the field.

\section{Immunofluorescence}

For immunofluorescence analysis of adherent cultures, cells were fixed with $4 \%$ paraformaldehyde (Millipore Sigma, Burlington, MA, USA) at room temperature for 15 min, washed three times with phosphate-buffered saline (PBS) and blocked with 5\% normal goat serum (Millipore) in PBS plus $0.1 \%$ Triton X-100 for $1 \mathrm{~h}$. Cells were incubated with an anti-N-cadherin (1:200; 13116S, Cell
Signaling) antibody. Cells were washed three times with PBS plus $0.1 \%$ Triton X-100 (Millipore) and incubated with appropriate secondary antibody (1:100 anti-mouse IgG3 Alexa 488 (\# A-21151) and 1:300 goat anti rabbit Alexa 568 (\# A-11011); Thermo Fisher Scientific). Nuclei were counterstained with DAPI solution, a coverslip added, and mounted on slides using VECTASHIELD Hardset mounting media (Vector Labs). Fluorescence micrographs were acquired using a Leica SP5 confocal microscope, and images were processed in Adobe Photoshop CS6 (Adobe).

The Drosophila strain slbo-GAL4, UAS-mCD8:GFP was crossed to $w^{1118}$ (control) or the following UAS-transgene flies to drive expression in border cells: UAS-Rap1 ${ }^{\mathrm{V} 12}$ (constitutively active Rap1), UAS-Cdc $42^{\mathrm{N} 17}$ (dominantnegative Cdc42), UAS-Cdc42 (wild-type Cdc42). Detailed information about Drosophila strains can be found in FlyBase (http://flybase.org/). Ovaries from the correct progeny of these crosses were dissected, fixed in $4 \%$ methanol-free formaldehyde (Polysciences) in phosphate buffer, and stained for anti-E-cadherin (DCAD2, Developmental Studies Hybridoma Bank (DSHB), University of Iowa) at 1:10 dilution using standard protocols ${ }^{67}$. Secondary antibodies used were anti-mouse (A-11004) or anti-rat Alexa 568 (A-11077) at 1:400 (Thermo Fisher Scientific). Nuclei were labeled using $0.05 \mu \mathrm{g} / \mathrm{ml}$ DAPI (Millipore). Ovaries were further dissected and mounted on slides in Aqua-PolyMount (Polysciences), followed by imaging on a Zeiss AxioImager Z1 microscope. Images were taken using a Zeiss Axiocam 503 camera, the Zeiss ApoTome 2 module, and a $20 \times 0.75$ NA objective, using either Zeiss AxioVision 4.8 or Zen software.

\section{Time-lapse microscopy of border cell migration in ex vivo cultured egg chambers}

Live imaging was performed essentially as previously described $^{68,69}$. Briefly, Drosophila egg chambers were dissected from whole ovaries and cultured in Schneider's insect medium (Thermo Fisher Scientific) supplemented with $10 \%$ fetal bovine serum (Millipore Sigma), $0.20 \mathrm{mg} /$ $\mathrm{mL}$ insulin (Millipore), and penicillin/streptomycin (Thermo Fisher Scientific). Egg chambers were transferred onto a 50 mm Lumox $^{\circledast}$ gas-permeable bottom dish (Sarstedt, Inc.), overlaid with a $22 \times 22 \mathrm{~mm}$ coverslip and the edges sealed with halocarbon oil 27 (Millipore Sigma) to prevent evaporation.

Widefield fluorescence $z$-stack images ( $5 z$-sections at $3 \mu \mathrm{m}$ apart) were captured every $2 \mathrm{~min}$ for the duration of border cell migration (about 3 to $4 \mathrm{~h}$ ). The Zeiss Colibri light source ( $25 \%$ blue LED at $250 \mathrm{msec}$ exposure, full frame ROI) was used to illuminate GFP, which was expressed in border cells using the GAL4-UAS system. Movies were created using the Zeiss Axiovision 4.8 "Inside 4D" software module or the ImageJ distribution 
Fiji (http://fiji.sc) ${ }^{70}$. For each time-lapse movie, concurrent $z$-sections in which the border cell cluster was in focus were merged. Image brightness and/or contrast were adjusted in Fiji.

Protrusions were defined manually, as in Sawant et al. ${ }^{53}$. Essentially, the converted QuickTime movies were analyzed using Fiji. Cellular extensions that were longer than 10 pixels (at least about $4 \mu \mathrm{m}$ ) from the main cell body were considered to be "protrusions." Only those protrusions that appeared within the first hour of each movie were tracked for analyses. Statistical tests were performed in GraphPad Prism 7.

\section{Time-lapse microscopy of tumor spheres}

Floating spheres were transferred into Geltrex-coated 6-well dishes. Live time-lapse movies of adherent CSC cells were acquired using a Leica DMI6000 inverted microscope and LAS X software v3.4.18368.2 (Leica Microsystems) equipped with a Hamamatsu ImageEM CCD camera and a Hamamatsu Orca Flash4 camera (Hamamatsu Photonics). Phase contrast images of multiple fields per well were collected every $15 \mathrm{~min}$ for $36 \mathrm{~h}$ using a $10 \times 0.4 \mathrm{NA}$ objective lens. Image processing and analysis was performed using the open source analysis program Fiji (64 bit, build 2014.11.25) ${ }^{70}$. Change in confluency of CSC cells over time was quantified for 3 different time points from each of the time-lapse movies as a measure of cell proliferation and migration. Selected phase contrast images were preprocessed by first converting them to 32 bit images and then using the Contrast Limited Adaptive Histogram Equalization (CLAHE) plugin to increase contrast and flatten image background. Image segmentation and quantification of confluency of the CLAHE processed phase contrast images was then completed using the PHANTAST plugin ${ }^{71}$.

\section{Immunoblotting}

Cell populations were lysed using NP-40 lysis buffer (containing PMSF, protease inhibitor cocktail, phosphatase inhibitor cocktail). Protein concentrations were calculated using a BCA protein assay (Pierce Biotechnology). After denaturation with Laemmli buffer (BioRad Laboratories), $20 \mathrm{mg}$ of total protein were loaded on $10 \%$ polyacrylamide SDS-PAGE gels, transferred to polyvinyl difluoride membranes (Millipore) and probed using the following antibodies: SOX2 (Cell Signaling, $2748 \mathrm{~S}$, 1:1000), Rapla (Abcam, ab115776, 1:1000) and B-Actin (Santa Cruz Biotechnology, sc-47778, 1:5000) was used as a loading control. Species-specific horseradish peroxidaseconjugated secondary antibodies were used for detection (Invitrogen, 1:5000). Membranes were developed using ECL-2 reagent (Pierce Biotechnology).

\section{Cell manipulations}

CSCs were transiently transfected in $10 \mathrm{~cm}$ plates. Once cells achieved a confluency of about $75 \%$, they were transfected with a preparation of OptiMEM, $14.37 \mu \mathrm{g}$ of DNA, and FuGENE HD Transfection reagent (Promega). On day 2 after the transfection, cells were removed from their plates with Accutase (BioLegend) and counted. For Rap1a alterations, CSCs were transfected with RAP1 WT, RAP1 N17, RAP1 63E, and with an eGFP control. Cdc42 was inhibited in T387 cells using ML 141 (Sigma) at $200 \mu \mathrm{M}$. Rap1 WT and N17 constructs were obtained from previously published sources ${ }^{72}$. Rap1 63E was obtained from Addgene (Plasmid \#32698) from previously published sources ${ }^{72,73}$. The eGFP plasmid was obtained from Lonza.

\section{Cell viability assays}

2000 cells were added to Geltrex-coated 96-well plates. Opaque clear bottom plates were used for both CellTiterGlo and CaspaseGlo (Promega) assays and regular cell culture plates were used for CyQUANT assay (Thermo Fisher Scientific). Cells were incubated at $37^{\circ} \mathrm{C}$ and $5 \%$ $\mathrm{CO}_{2}$ for the duration of the experiment. Assays were completed according to manufacturer's directions on 1- and 3-days post-plating or addition of the inhibitor. CaspaseGlo measurements were normalized to CellTiterGlo measurements.

\section{Statistics}

All statistical analyses were performed in GraphPad Prism 5.0 (GraphPad Software). Unless otherwise indicated, a student's $t$ test or one-way ANOVA was used and a $p$ value of $\leq 0.05$ was considered significant.

\section{Acknowledgements \\ This work used the Leica SP5 confocal/multi-photon microscope that was purchased with partial funding from National Institutes of Health SIG grant 1S10RR026820-01. This work was supported in part by a fellowship from the Kansas INBRE through the National Institutes of Health grant P20 GM103418 to Y.C., NIH R21 CA198254 to J.D.L. and J.A.M., and support from the Cleveland Clinic (including the Brain Tumor Research and Therapeutic Development Center of Excellence) and Case Comprehensive Cancer Center to J.D.L.}

\section{Author details \\ 'Lerner Research Institute, Cleveland Clinic, Cleveland, OH 44195, USA. ${ }^{2}$ Cleveland Clinic Lerner College of Medicine of Case Western Reserve University, Cleveland, OH 44195, USA. ${ }^{3}$ Division of Biology, Kansas State University, Manhattan, KS 66506, USA. ${ }^{4}$ Department of Nutrition, Case Western Reserve University School of Medicine, Cleveland, OH 44106, USA. ${ }^{5}$ Case Comprehensive Cancer Center, Case Western Reserve University, Cleveland, $\mathrm{OH}$ 44106, USA. 'Present address: Division of Immunity and Pathogenesis, Burnett School of Biomedical Sciences, University of Central Florida College of Medicine, Orlando, FL, USA}

Conflict of interest

The authors declare that they have no conflict of interest. 


\section{Publisher's note}

Springer Nature remains neutral with regard to jurisdictional claims in published maps and institutional affiliations.

Supplementary Information accompanies this paper at (https://doi.org/ 10.1038/s41419-020-2342-2).

Received: 17 June 2019 Revised: 3 December 2019 Accepted: 4 December 2019

Published online: 26 February 2020

\section{References}

1. Stupp, R. et al. Effects of radiotherapy with concomitant and adjuvant temozolomide versus radiotherapy alone on survival in glioblastoma in a randomised phase III study: 5-year analysis of the EORTC-NCIC trial. Lancet Oncol. 10, 459-466 (2009).

2. Stupp, R. et al. Radiotherapy plus concomitant and adjuvant temozolomide for glioblastoma. N. Engl. J. Med. 352, 987-996 (2005).

3. Stupp, R. et al. Effect of tumor-treating fields plus maintenance temozolomide vs maintenance temozolomide alone on survival in patients with glioblastoma: a randomized clinical trial. JAMA: J. Am. Med. Assoc. 318, 2306-2316 (2017).

4. Ostrom, Q. T. et al. CBTRUS Statistical Report: primary brain and other central nervous system tumors diagnosed in the United States in 2010-2014. NeuroOncology 19, v1-v88 (2017).

5. Bao, S. et al. Glioma stem cells promote radioresistance by preferential activation of the DNA damage response. Nature 444, 756-760 (2006).

6. Chen, J. et al. A restricted cell population propagates glioblastoma growth after chemotherapy. Nature 488, 522-526 (2012).

7. $\mathrm{Yu}, \mathrm{S}$. P. et al. Enhanced invasion in vitro and the distribution patterns in vivo of CD133+ glioma stem cells. Chin. Med. J. 124, 2599-2604 (2011).

8. Inoue, A. et al. Cancer stem-like cells of glioblastoma characteristically express MMP-13 and display highly invasive activity. Int. J. Oncol. 37 1121-1131 (2010).

9. Cheng, L. et al. Elevated invasive potential of glioblastoma stem cells. Biochem. Biophys. Res. Commun. 406, 643-648 (2011).

10. Mehta, S. \& Lo Cascio, C. Developmentally regulated signaling pathways in glioma invasion. Cell. Mol. Life Sci.: CMLS 75, 385-402 (2018).

11. Visvader, J. E. \& Lindeman, G. J. Cancer stem cells: current status and evolving complexities. Cell Stem Cell 10, 717-728 (2012)

12. Paez-Ribes, M. et al. Antiangiogenic therapy elicits malignant progression of tumors to increased local invasion and distant metastasis. Cancer Cell 15 220-231 (2009).

13. Lu, K. V. et al. VEGF inhibits tumor cell invasion and mesenchymal transition through a METNEGFR2 complex. Cancer Cell 22, 21-35 (2012).

14. Cuddapah, V. A., Robel, S., Watkins, S. \& Sontheimer, H. A neurocentric perspective on glioma invasion. Nat. Rev. Neurosci. 15, 455-465 (2014).

15. Winkler, F. et al. Imaging glioma cell invasion in vivo reveals mechanisms of dissemination and peritumoral angiogenesis. Glia 57, 1306-1315 (2009).

16. Farin, A. et al. Transplanted glioma cells migrate and proliferate on host brain vasculature: a dynamic analysis. Glia 53, 799-808 (2006).

17. Friedl, P., Locker, J., Sahai, E. \& Segall, J. E. Classifying collective cancer cell invasion. Nat. Cell Biol. 14, 777-783 (2012).

18. Wang, X., Enomoto, A., Asai, N., Kato, T. \& Takahashi, M. Collective invasion of cancer: perspectives from pathology and development. Pathol. Int. 66 183-192 (2016)

19. Ilina, O. et al. Intravital microscopy of collective invasion plasticity in breast cancer. Dis. Models Mech. 11, https://doi.org/10.1242/dmm.034330 (2018).

20. Khalil, A. A. et al. Collective invasion in ductal and lobular breast cancer associates with distant metastasis. Clin. Exp. Metastasis 34, 421-429 (2017).

21. Cheung, K. J. \& Ewald, A. J. A collective route to metastasis: seeding by tumor cell clusters. Science 352, 167-169 (2016).

22. Bronsert, P. et al. Cancer cell invasion and EMT marker expression: a threedimensional study of the human cancer-host interface. J. Pathol. 234, 410-422 (2014).

23. Kim, Y. H. et al. Senescent tumor cells lead the collective invasion in thyroid cancer. Nat. Commun. 8, 15208 (2017).

24. Hou, J. M. et al. Circulating tumor cells as a window on metastasis biology in lung cancer. Am. J. Pathol. 178, 989-996 (2011).
25. Te Boekhorst, V., Preziosi, L. \& Friedl, P. Plasticity of cell migration in vivo and in silico. Annu. Rev. Cell Dev. Biol. 32, 491-526 (2016).

26. Gritsenko, P., Leenders, W. \& Friedl, P. Recapitulating in vivo-like plasticity of glioma cell invasion along blood vessels and in astrocyte-rich stroma. Histochem. Cell Biol. 148, 395-406 (2017).

27. Gritsenko, P. G. \& Friedl, P. Adaptive adhesion systems mediate glioma cell invasion in complex environments. J. Cell Sci. 131, jcs.216382 (2018).

28. Scarpa, E. \& Mayor, R. Collective cell migration in development. J. Cell Biol. 212, 143-155 (2016).

29. Friedl, P. \& Gilmour, D. Collective cell migration in morphogenesis, regeneration and cancer. Nat. Rev. Mol. Cell Biol. 10, 445-457 (2009).

30. Montell, D. J., Yoon, W. H. \& Starz-Gaiano, M. Group choreography: mechanisms orchestrating the collective movement of border cells. Nat. Rev. Mol. Cell Biol. 13, 631-645 (2012).

31. Stuelten, C. H., Parent, C. A. \& Montell, D. J. Cell motility in cancer invasion and metastasis: insights from simple model organisms. Nat. Rev. Cancer 18, 296-312 (2018).

32. Yoshida, $\mathrm{H}$. et al. Lessons from border cell migration in the Drosophila ovary: a role for myosin VI in dissemination of human ovarian cancer. Proc. Natl Acad. Sci. USA 101, 8144-8149 (2004).

33. Chen, $X$. et al. A feedforward mechanism mediated by mechanosensitive ion channel PIEZO1 and tissue mechanics promotes glioma aggression. Neuron 100, 799-815, e797 (2018)

34. Read, R. D. et al. A kinome-wide RNAi screen in Drosophila Glia reveals that the RIO kinases mediate cell proliferation and survival through TORC2-Akt signaling in glioblastoma. PLoS Genet. 9, e1003253 (2013).

35. Mukherjee, S. et al. Drosophila Brat and human ortholog TRIM3 maintain stem cell equilibrium and suppress brain tumorigenesis by attenuating notch nuclear transport. Cancer Res. 76, 2443-2452 (2016).

36. Goswami, S. et al. Macrophages promote the invasion of breast carcinoma cells via a colony-stimulating factor-1/epidermal growth factor paracrine loop. Cancer Res. 65, 5278-5283 (2005)

37. Saadin, A. \& Starz-Gaiano, M. Circuitous genetic regulation governs straightforward cell migration. Trends Genet.: TIG 32, 660-673 (2016).

38. Naora, H. \& Montell, D. J. Ovarian cancer metastasis: integrating insights from disparate model organisms. Nat. Rev. Cancer 5, 355-366 (2005).

39. Prasad, M. \& Montell, D. J. Cellular and molecular mechanisms of border cell migration analyzed using time-lapse live-cell imaging. Dev. Cell 12, 997-1005 (2007).

40. Aranjuez, G., Kudlaty, E., Longworth, M. S. \& McDonald, J. A. On the role of PDZ domain-encoding genes in Drosophila border cell migration. G3 2, 1379-1391 (2012).

41. Hu, Y. et al. An integrative approach to ortholog prediction for disease-focused and other functional studies. BMC Bioinform. 12, 357 (2011).

42. Garcia-Mata, R. \& Burridge, K. Catching a GEF by its tail. Trends Cell Biol. 17, 36-43 (2007).

43. Zegers, M. M. \& Friedl, P. Rho GTPases in collective cell migration. Small GTPases 5, e28997 (2014).

44. Combedazou, A. et al. Small GTPases orchestrate cell-cell communication during collective cell movement. Small GTPases, 11, 103-112 (2020).

45. Prunier, C., Prudent, R., Kapur, R., Sadoul, K. \& Lafanechere, L. LIM kinases: cofilin and beyond. Oncotarget 8, 41749-41763 (2017).

46. Hall, A. Rho family GTPases. Biochemical Soc. Trans. 40, 1378-1382 (2012).

47. Tepass, U. The apical polarity protein network in Drosophila epithelial cells: regulation of polarity, junctions, morphogenesis, cell growth, and survival. Annu. Rev. Cell Dev. Biol. 28, 655-685 (2012).

48. Ridley, A. J. Rho GTPase signalling in cell migration. Curr. Opin. Cell Biol. 36, 103-112 (2015).

49. Etienne-Manneville, S., Manneville, J. B., Nicholls, S., Ferenczi, M. A. \& Hall, A. Cdc42 and Par6-PKCzeta regulate the spatially localized association of Dlg1 and APC to control cell polarization. J. Cell Biol. 170, 895-901 (2005)

50. Okura, $\mathrm{H}$. et al. A role for activated Cdc42 in glioblastoma multiforme invasion Oncotarget 7, 56958-56975 (2016).

51. Colombie, N. et al. Non-autonomous role of Cdc42 in cell-cell communication during collective migration. Dev. Biol. 423, 12-18 (2017).

52. Cordero, D. et al. Small molecule inhibitors limit endothelial cell invasion by Staphylococcus aureus. Curr. Pharm. Biotechnol. 15, 727-737 (2014).

53. Sawant, K., Chen, Y., Kotian, N., Preuss, K. M. \& McDonald, J. A. Rap1 GTPase promotes coordinated collective cell migration in vivo. Mol. Biol. Cell 29 2656-2673 (2018) 
54. Chang, Y. C. et al. Rap1 negatively regulates the hippo pathway to polarize directional protrusions in collective cell. Migr. Cell Rep. 22, 2160-2175 (2018).

55. Te Boekhorst, V. \& Friedl, P. Plasticity of cancer cell invasion-mechanisms and implications for therapy. Adv. Cancer Res. 132, 209-264 (2016).

56. Prasad, M., Wang, X., He, L., Cai, D. \& Montell, D. J. Border cell migration: a model system for live imaging and genetic analysis of collective cell movement. Methods Mol. Biol. 1328, 89-97 (2015).

57. Subbaiah, V. K., Kranjec, C., Thomas, M. \& Banks, L. PDZ domains: the building blocks regulating tumorigenesis. Biochem. J. 439, 195-205 (2011).

58. Saito, Y., Desai, R. R. \& Muthuswamy, S. K. Reinterpreting polarity and cancer: the changing landscape from tumor suppression to tumor promotion. Biochim. Biophys. Acta. Rev. Cancer 1869, 103-116 (2018).

59. Gandalovicova, A., Vomastek, T., Rosel, D. \& Brabek, J. Cell polarity signaling in the plasticity of cancer cell invasiveness. Oncotarget 7, 25022-25049 (2016).

60. Xie, Q., Mittal, S. \& Berens, M. E. Targeting adaptive glioblastoma: an overview of proliferation and invasion. Neuro-Oncology 16, 1575-1584 (2014).

61. Zhang, Y. L., Wang, R. C., Cheng, K., Ring, B. Z. \& Su, L. Roles of Rap1 signaling in tumor cell migration and invasion. Cancer Biol. Med. 14, 90-99 (2017).

62. Frische, E. W. \& Zwartkruis, F. J. Rap1, a mercenary among the Ras-like GTPases. Dev. Biol. 340, 1-9 (2010).

63. Barrett, A. et al. A crucial role for DOK1 in PDGF-BB-stimulated glioma cell invasion through p130Cas and Rap1 signalling. J. Cell Sci. 127, 2647-2658 (2014).
64. Sayyah, J. et al. The Ras-related protein, Rap1A, mediates thrombin-stimulated integrin-dependent glioblastoma cell proliferation and tumor growth. J. Biol. Chem. 289, 17689-17698 (2014).

65. Alvarado, A. G. et al. Coordination of self-renewal in glioblastoma by integration of adhesion and microRNA signaling. Neuro-Oncology 18, 656-666 (2016).

66. Lathia, J. D. et al. High-throughput flow cytometry screening reveals a role for junctional adhesion molecule a as a cancer stem cell maintenance factor. Cell Rep. 6, 117-129 (2014)

67. McDonald, J. A. \& Montell, D. J. Analysis of cell migration using Drosophila as a model system. Methods Mol. Biol. 294, 175-202 (2005).

68. Prasad, M., Jang, A. C., Starz-Gaiano, M., Melani, M. \& Montell, D. J. A protoco for culturing Drosophila melanogaster stage 9 egg chambers for live imaging. Nat. Protoc. 2, 2467-2473 (2007).

69. Majumder, P., Aranjuez, G., Amick, J. \& McDonald, J. A. Par-1 controls myosin-\| activity through myosin phosphatase to regulate border cell migration. Curr. Biol. 22, 363-372 (2012).

70. Schindelin, J. et al. Fiji: an open-source platform for biological-image analysis. Nat. Methods 9, 676-682 (2012).

71. Jaccard, N. et al. Automated method for the rapid and precise estimation of adherent cell culture characteristics from phase contrast microscopy images. Biotechnol. Bioeng. 111, 504-517 (2014).

72. Kitayama, H., Matsuzaki, T., Ikawa, Y. \& Noda, M. Genetic analysis of the Kirstenras-revertant 1 gene: potentiation of its tumor suppressor activity by specific point mutations. Proc. Natl Acad. Sci. USA 87, 4284-4288 (1990).

73. Lafuente, E. M. et al. RIAM, an EnaNASP and Profilin ligand, interacts with Rap1-GTP and mediates Rap1-induced adhesion. Dev. Cell 7, 585-595 (2004). 